\title{
Compensation of Finite Bandwidth Effect by Using an Optimal Filter in Photoacoustic Imaging*
}

\author{
Chen Zhang, Yan Zhang, Yuanyuan Wang \\ Department of Electronic Engineering, Fudan University, Shanghai, China \\ Email: 11110720018@fudan.edu.cn,yywang@fudan.edu.cn
}

Received September 2012

\begin{abstract}
Most existing reconstruction algorithms for photoacoustic imaging (PAI) assume that transducers used to receive ultrasound signals have infinite bandwidth. When transducers with finite bandwidth are used, this assumption may result in reduction of the imaging contrast and distortions of reconstructed images. In this paper, we propose a novel method to compensate the finite bandwidth effect in PAI by using an optimal filter in the Fourier domain. Simulation results demonstrate that the use of this method can improve the contrast of the reconstructed images with finite-bandwidth ultrasound transducers.
\end{abstract}

Keywords: Photoacoustic Tomography; Finite Bandwidth Effect; Optimal Filter

\section{Introduction}

Photoacoustic imaging (PAI) is a promising medical imaging modality that has great potential for a wide range of clinical imaging applications [1,2]. PAI is a hybrid imaging method that combines the high contrast of optical imaging and the high spatial resolution of ultrasonic imaging. Due to its noninvasive nature, it is a technique which is harmless for the human body [2].

In PAI, pulsed laser energy is delivered to an object and the thermoacoustic effect will result in the generation of the ultrasound wave. Ultrasound signals are then detected by the scanning ultrasound transducers. With these received signals, an image reconstruction algorithm is used to reconstruct the image of an object's optical absorption distribution.

A variety of research has been done on the field of image reconstruction algorithms [3-10]. Most of these algorithms are based on the assumption that infinitebandwidth transducers are employed to receive ultrasound signals. Without incorporating characteristics of ultrasound transducers in the imaging reconstruction model, the resolution and the contrast of reconstructed images will be significantly degraded. Several imaging models have been established to incorporate physical characteristics of ultrasound transducers [11,12]. However, none of them takes the finite bandwidth effect of ultrasound transducers into account.

*Natural Science Foundation of China (No. 61271071 and No. 11228411) and Specialized Research Fund forthe Doctoral Program of Higher Education of China (No. 20110071110017).
In this paper, we propose a novel image reconstruction algorithm that incorporates the finite bandwidth characteristics of ultrasound transducers. An optimal filter is designed to deconvolve the transducer's impulse response of the finite bandwidth at each imaging point. Through the numerical simulation, the proposed algorithm is compared to those reconstruction algorithms assuming finite-bandwidth ultrasound transducers.

\section{Theory and Method}

\subsection{Basic Principles of PAI}

In this study, we intend to solve the problem with 2D circular-scanning PAI mode. In this mode, a short pulse is used to illuminate the imaging object from the top, and an ultrasound transducer is circularly scanned around the object to record the ultrasound data. The relation between the optical absorption distribution and the generated ultrasonic waves can be deduced as [3]:

$$
\nabla^{2} p(\mathbf{r}, t)-\frac{1}{c^{2}} \frac{\partial^{2} p(\mathbf{r}, t)}{\partial t^{2}}=-\frac{\beta}{C_{p}} A(\mathbf{r}) \frac{\partial I(t)}{\partial t},
$$

where $c$ is the speed of sound, $\beta$ is the volume thermal expansion coefficient and $C_{p}$ is the specific heat. $A(\mathbf{r})$ is the unknown absorption distribution and $I(\mathrm{t})$ is the illumination function. A solution to this equation can be obtained by the use of Green's function. In the case of illumination with a short pulse, $p(\mathbf{r}, t)$ can be deduced as:

$$
p\left(\mathbf{r}_{0}, t\right)=\frac{\beta}{4 \pi C_{p}} \frac{\partial}{\partial t} \oiint_{\left|\mathbf{r}^{\prime} \mathbf{r}_{0}\right|=c t} \frac{A\left(\mathbf{r}^{\prime}\right)}{t} d^{2} \mathbf{r}^{\prime}
$$

Equation (2) represents an idealized imaging model for 
PAI. The image reconstruction problem is to determine an estimate of $A(\mathbf{r})$ from the knowledge of $p(\mathbf{r}, t)$. A variety of image reconstruction algorithms [3-10] has been developed for the inversion of (2). However, those algorithms neglect the response characteristics of the transducer. Therefore they may produce significant image blurring and low contrast in the reconstructed images.

\subsection{Finite Bandwidth Effect of a Transducer}

Most of PAI reconstruction algorithms are based on the assumption that the bandwidth of ultrasonic transducers which are employed to receive signals is infinite. However, actual ultrasound transducers are all with the finite bandwidth. The finite bandwidth effect of transducers can be expressed as a convolution of received signals and an impulse response. The actual projection data can be expressed as:

$$
p\left(r_{0}, t\right)=p^{\text {ideal }}\left(r_{0}, t\right) * h(r, t),
$$

where the $p^{\text {ideal }}\left(\boldsymbol{r}_{0}, t\right)$ is the idealized projection data based on the assumption that the transducers have the infinite bandwidth, $p\left(\boldsymbol{r}_{\mathbf{0}}, t\right)$ is the actual projection data obtained by the transducers, $h\left(\boldsymbol{r}_{\mathbf{0}}, t\right)$ is the impulse response of the transducer related to its bandwidth, * denotes a one-dimensional temporal convolution.

Figure 1 compares ultrasonic signals obtained by an idealized transducer and a finite-bandwidth transducer. Ultrasonic signals are excited by a point optical absorber. Figure 1 illustrated that the finite-bandwidth effect may cause the reduction of the signal density and the convolutional noise. These are major reasons of image blurring and low contrast in reconstructed images if image reconstruction algorithms fail to take the finite-bandwidth effect into account.

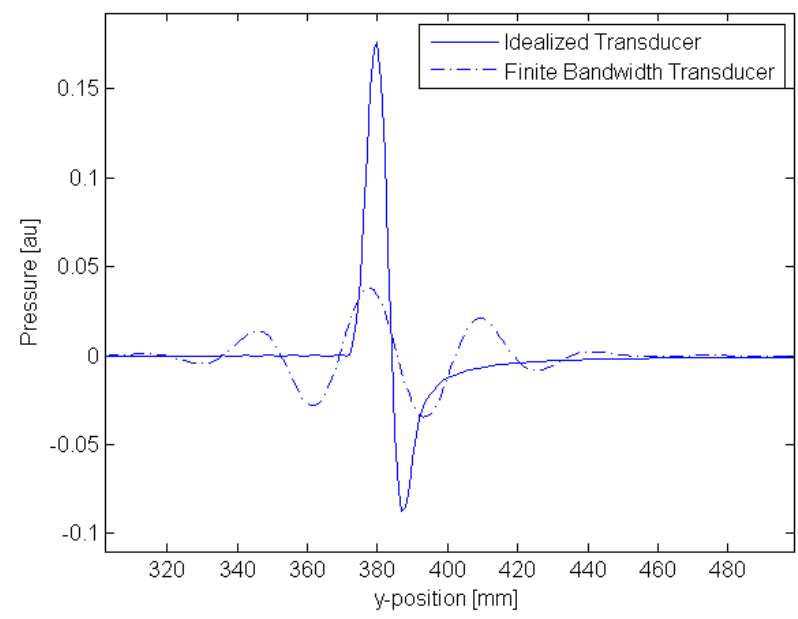

Figure 1. A comparison between ultrasound signals obtained by an idealized transducer and a finite-bandwidth transducer.

\subsection{Compensating for the Finite Bandwidth Effect}

Based on (3), the purpose of compensation for the finite bandwidth effect is to obtain the $p^{\text {ideal }}\left(\boldsymbol{r}_{0}, t\right)$. As the received signals are non-stationary signals, we use the short time Fourier transform (STFT) to transform the data into the Fourier domain. Here we take the additive noise into account. Equation (3) is transformed as:

$$
P\left(r_{0}, \omega\right)=P^{\text {ideal }}\left(r_{0}, \omega\right) \cdot H(r, \omega)+e
$$

where $e$ is the additive noise, $H(\mathbf{r}, \omega)$ is the bandwidth of the transducer, $P\left(\mathbf{r}_{\mathbf{0}}, \omega\right)$ and $P^{\text {ideal }}\left(\mathbf{r}_{\mathbf{0}}, \omega\right)$ is the Fourier spectrum of the actual projection data and the idealized data.

An optimal filter $\boldsymbol{T}$ can be derived to obtain received signals where the finite bandwidth effect is compensated.

$$
P^{\text {ideal }}\left(r_{0}, \omega\right)=T \cdot P\left(r_{0}, \omega\right)
$$

In this study, this optimal filter $\boldsymbol{T}$ is designed to minimize the error between $P\left(\mathbf{r}_{\mathbf{0}}, \omega\right)$ and $P^{\text {ideal }}\left(\mathbf{r}_{\mathbf{0}}, \omega\right)$. At the same time, the optimal filter should have good robustness against the additive noise. We can easily get the bandwidth characteristics of a transducer from its data sheet or from the physical meansurement experiment. In the Fourier domain, its distribution function is Gaussian. The optimal filter $\boldsymbol{T}$ can be found:

$$
T=1 /\left(1+\phi /\left|\Delta^{2}\right|\right)
$$

where $\varphi$ is a parameter determine the noise suppression effect and $\Delta$ a Gaussian kernel function which can be expressed as:

$$
\Delta=\frac{1}{\sigma \sqrt{2 \pi}} e^{-\frac{(t-\mu)}{2 \sigma^{2}}} .
$$

Here $\mu$ is the mean of the function, $\sigma$ is the variance of the function. Both of those parameters can be obtained from the bandwidth characteristics of the transducer.

Once we obtained the actual projection data, we can reconstruct the image with the compensated data.

\section{Results and Discussion}

Simulations are performed to evaluate the efficacy of this method on the improvement of resolution and contrast of the reconstructed image. The comparison are made among this method and those reconstruction algorithms assuming finite-bandwidth ultrasound transducers. All simulations are done using the K-wave toolbox $[13,14]$ of MATLAB.

In this simulation, three optical absorbers with different radius and optical absorption density are located in the test image. Its original optical energy distribution is showed in Figure 2. We set the radius of scanning circle as 250 pixels and assume the speed of sound consistent 


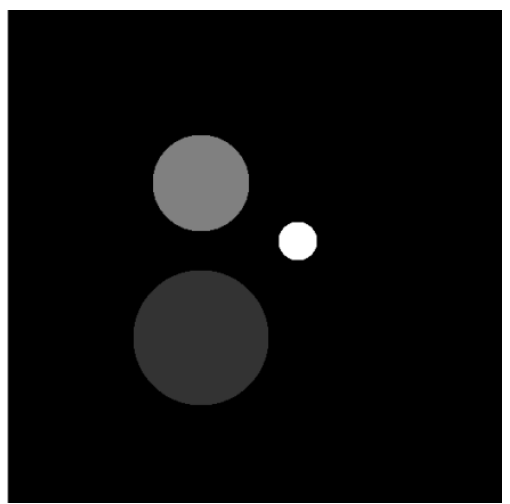

Figure 2. The original optical energy distribution of the simulation image.

as $1500 \mathrm{~m} / \mathrm{s}$. The simulated transducer has a center frequency of $5 \mathrm{MHz}$ with $80 \%$ bandwidth. 180 scanning angels in 360-degree full view are used to obtain the projection data.

\subsection{Results from Noiseless Simulation Data}

The computer-simulated noiseless data are used to evaluate the efficacy of this method. The image reconstructed without compensation is shown in Figure 3(a) while the image reconstructed by using this method is shown in Figure 3(b).

As expected, the simulation results show that the image reconstructed with compensated data has improved the contrast compared with the image reconstructed without compensation. Figure 3(a) contains negative values and is shown in a different gray scale from that employed in Figure 3(b). The profiles along the radial directions are shown in Figure $\mathbf{4}$ in order to compare the detail qualities of reconstructed images clearly. In Figure 4, the solid and dashed lines represent pixel profiles of the reconstructed image without compensation and after compensation respectively. It clearly shows that the contrast of the reconstructed image after compensation is better than that without compensation.

The peak signal-to-noise ratios (PSNRs) of reconstructed images are adopted with the original image as the standard in order to provide the numeric quantification of results. PSNR is defined as:

$$
P S N R=10 \lg \frac{N_{x} N_{y}}{\sum_{i=1}^{N_{x}} \sum_{j=1}^{N_{y}}\left(f_{i, j}-r_{i, j}\right)^{2}}
$$

where $f$ is the pixel-value of the reconstructed image and $r$ is the pixel-value of the original image. The size of the image is $N_{x} N_{y}$.

The PSNR value of the reconstructed image with compensation is 50.18 while that without compensation is 17.33. This result demonstrates that the reconstructed image after compensation is closer to the original image.

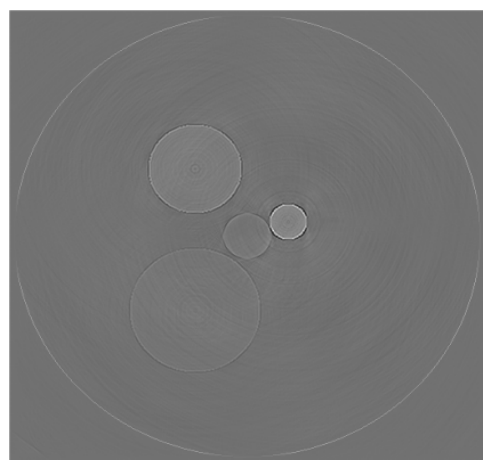

(a)

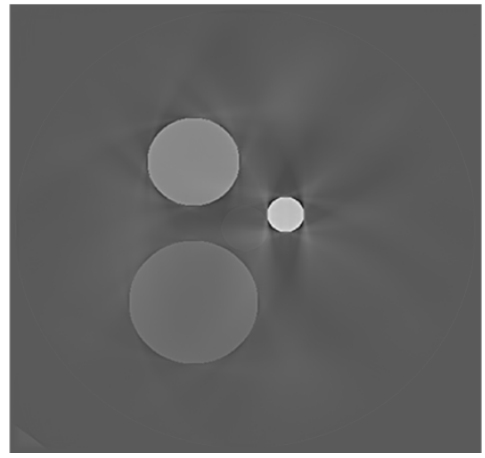

(b)

Figure 3. Images reconstructed from the noiseless data (a) without compensation (b) after compensation.

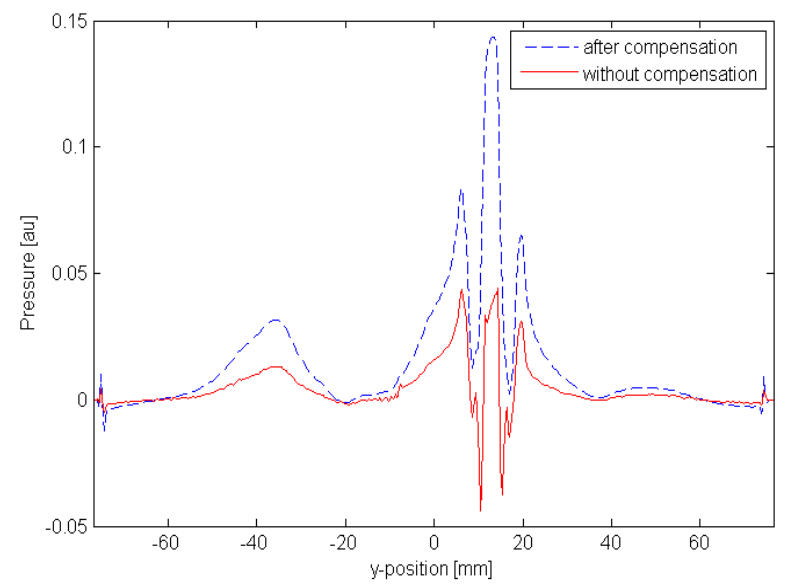

Figure 4. The radial pixel profiles of Figure 3.

\subsection{Results from Noisy Simulation Data}

We simulate the noisy data by adding the white noise to received signals. This simulation is performed to evaluate the robustness of this method against the additive noise. The image with the background noise is shown in Figure 5. The image reconstructed without compensation is shown in Figure 6(a) while the image reconstructed by using this method is shown in Figure 6(b).

The result clearly shows that this method can also improve the contrast of the reconstructed image with the 


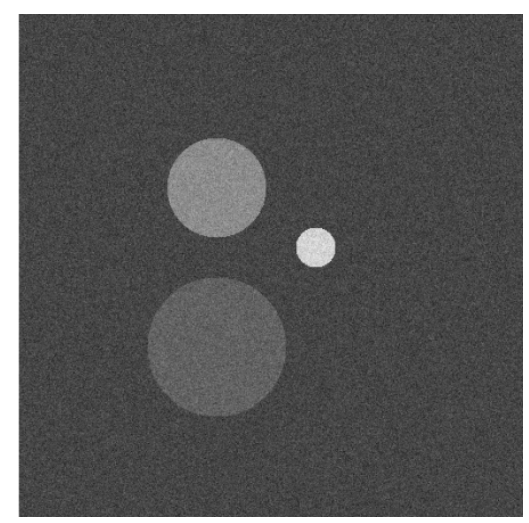

Figure 5. The simulation image with the background noise.

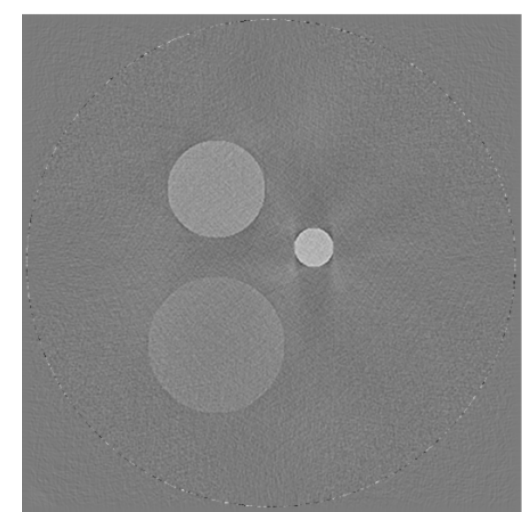

(a)

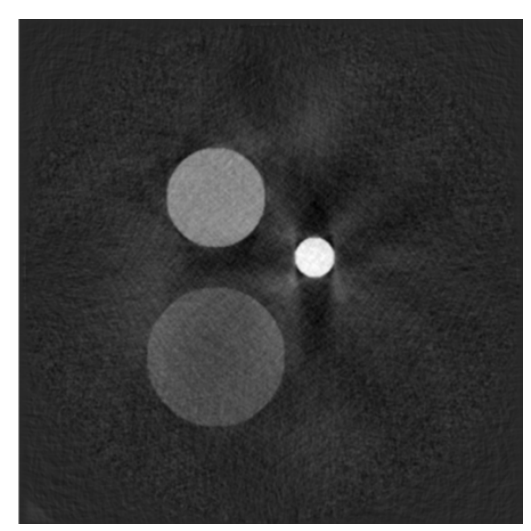

(b)

Figure 6. The images reconstructed from the noisy data (a) without compensation (b)after compensation.

noisy data. This seems to be a robust algorithm. The profiles along the radial directions are shown in Figure 7. In Figure 7, the solid and dashed lines represent the pixel profiles of the reconstructed image without compensation and after compensation respectively. It clearly shows that the contrast of the reconstructed image after compensation is better than that without compensation. It is also seen that the density of the noise is weaker than that without compensation. Through this simulation, the result shows that this method can reconstruct images with

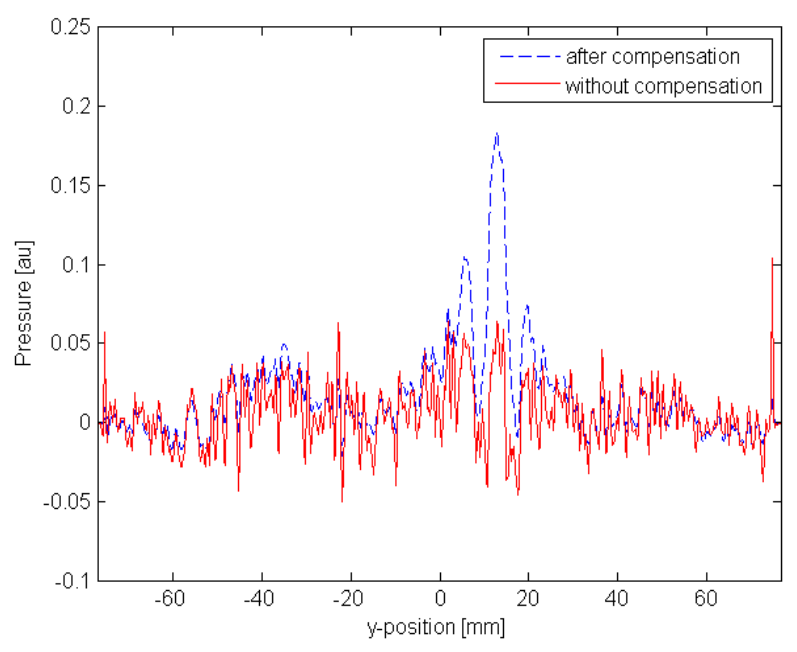

Figure 7. The radical pixel profiles of Figure 6.

lower noise levels, meanwhile the algorithm is robust.

\section{Conclusion}

In this paper, we propose a method to compensate the finite bandwidth effect of the ultrasound transducer. It is seen from simulation studies that the proposed method has good robustness and can effectively improve the image contrast for PAI with finite-bandwidth transducers.

\section{REFERENCES}

[1] L. V. Wang, "Tutorial on Photoacoustic Microscopy and Computed Tomography,” IEEE Journal of Selected Topics Quantum Electronics, Vol. 14, No. 1, 2008, pp. 171179. http://dx.doi.org/10.1109/JSTQE.2007.913398

[2] C. Li and L. V. Wang, "Photoacoustic Tomography and Sensing in Biomedicine," Physics in Medicine and Biology, Vol. 54, No. 19, 2009, pp. R59-R97. http://dx.doi.org/10.1088/0031-9155/54/19/R01

[3] M. Xu and L. V. Wang, "Universal Back-Projection Algorithm for Photacoustic Computed Tomography,” Physical Review E, Vol. 71, No. 1, Article ID: 016706. http://dx.doi.org/10.1103/PhysRevE.71.016706

[4] P. Burgholzer, J. Bauer-Marschallinger, H. Grun, M. Haltmeier and G. Paltauf, "Temporal Back-Projection Algorithms for Photoacoustic Tomography with Integrating Line Detectors,” Inverse Problems, Vol. 23, No. 6, 2007, pp. S65-S80.

http://dx.doi.org/10.1088/0266-5611/23/6/S06

[5] M. Xu, Y. Xu and L. V. Wang, "Time-Domain Reconstruction Algorithms and Numerical Simulations for Thermoacoustic Tomography in Various Geometries," IEEE Transactions on Biomedical Engineering, Vol. 50, No. 9, 2003, pp. 1086-1099. http://dx.doi.org/10.1109/TBME.2003.816081

[6] Y. Xu, D. Feng and L. V.Wang, "Exact Frequency-Domain Reconstruction for Thermoacoustic Tomography: I. Planar Geometry,” IEEE Transactions on Medical Imag- 
ing, Vol. 21, No. 7, 2002, pp. 823-828. http://dx.doi.org/10.1109/TMI.2002.801172

[7] L. A. Kunyansky, "Explicit Inversion Formulae for the Spherical Mean Radon Transform,” Inverse Problems, Vol. 23, 2007, pp. 373-383. http://dx.doi.org/10.1088/0266-5611/23/1/021

[8] M. A. Anastasio, J. Zhang, X. Pan, Y. Zou, G. Keng and L. V. Wang, "Half-Time Image Reconstruction in Thermoacoustic Tomography," IEEE Transactions on Medical Imaging, Vol. 24, No. 2, 2005, pp. 199-210. http://dx.doi.org/10.1109/TMI.2004.839682

[9] P. Ephrat, L. Keenliside, A. Seabrook, F. S. Prato and J. J. L. Carson, "Three-Dimensional Photoacoustic Imaging by Sparse-Array Detection and Iterative Image Reconstruction,” Journal of Biomedical Optics, Vol. 13, No. 5, 2008. http://dx.doi.org/10.1121/1.1501898

[10] G. Paltauf, J. A. Viator, S. A. Prahl and S. L. Jacques, "Iterative Reconstructional Gorithm for Optoacoustic Imaging," Journal of the Acoustical Society of America, Vol. 112, No. 4, 2002, pp. 1536-1544. http://dx.doi.org/10.1121/1.1501898
[11] K. Wang, S. A. Ermilov, R. Su, H. Brecht, A. A. Oraevsky and M. A. Anastasio, "An Imaging Model Incorporating Ultrasonic Tranducer Properties for ThreeDimensional Optoacoustic Tomography,” IEEE Transactions on Medical Imaging, Vol. 30, No. 2, 2011, pp. 203213. http://dx.doi.org/10.1109/TMI.2010.2072514

[12] M. Li, Y. Tseng and C. Cheng, "Model-Based Correction of Finite Aperture Effect in Photoacoustic Tomography," Optics Express, Vol. 18, No. 25, 2010. http://dx.doi.org/10.1364/OE.18.026285

[13] B. T. Cox, S. Kara, S. R. Arridge and P. C. Beard, "k-Space Propagation Models for Acoustically Heterogeneous Media: Application to Biomedical Photoacoustics," Journal of the Acoustical Society of America, Vol. 121, No. 6, 2007, pp. 3453-3464. http://dx.doi.org/10.1121/1.2717409

[14] B. E. Treeby and B. T. Cox, "k-Wave: MATLAB Toolbox for the Simulation and Reconstruction of Photoacoustic Wave Fields,” Journal of Biomedical Optics, Vol. 15, No. 2, 2010. http://dx.doi.org/10.1117/1.3360308 